\title{
Small pustules (pin-sized) distributed on the lateral aspects of the face
}

\section{Krostki (wielkości łebka szpilki) zlokalizowane na bocznych powierzchniach twarzy}

Przegl Dermatol 2014, 101, 225-226

DOI: 10.5 | |4/dr.2014.438|5

We would like to present two cases of sterile pustules on the face, observed in young patients.

\section{CASE REPORTS}

\section{Case I}

A 23-year-old female patient was seen in the Dermatology Department for small pustules (pin-sized), without a rash, distributed on the lateral aspects of the face, observed 2 days prior to admission (Fig. 1). She was in a good state of health, with no history of dermatological diseases, and no history of drug intake. She was a student, not working with irritants or oily substances.

Usual laboratory investigations revealed no abnormal values. Direct mycological examination and culture for fungi were negative and culture from a pustule failed repeatedly to isolate any bacteria. Swabs taken from the nose and oral cavity were sterile. Endocrinological examination was normal.

\section{Case 2}

A 17-year-old man, a pupil, came to the Dermatology Unit due to sudden onset of a peculiar eruption around the mouth: small pustules with no erythematous base, non-follicular and non-pruritic (Fig. 2). He was otherwise a healthy person, with no chronic diseases and no previous treatments. The patient denied

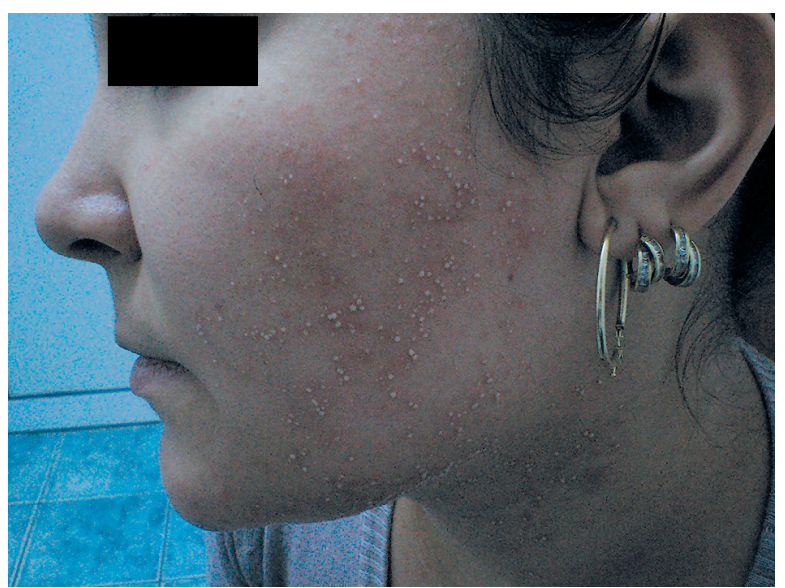

Figure I. Patient I. Small pustules (pin-sized) distributed on the lateral aspects of the face fever or other signs of infectious disease before the eruption. At clinical examination pin-sized pustules distributed in clusters, non-flaccid, non-tender, with no signs of inflammation were seen at the margins of the lips and on the menton. A few similar pustules were present on the lips too. No adenopathy, no fever, and no other dermatological lesions were observed.

Results of complete blood count with differential leucocyte count were unremarkable. Gram stain of blister fluid was negative. Analysis of blister fluid with $10 \%$ potassium hydroxide preparation showed no fungal elements. The Tzanck test was negative. HIV and VDRL tests were negative. Full blood count, coagulation screen, serum chemistry and liver function tests were all within normal limits.

\section{DISCUSSION}

All pustules in patients were parafollicular. Previously, they did not use cosmetics with heavy oil as make-up. There are many similarities between the cases: young persons during educational period (a student and a pupil); healthy people, no history of drug intake; pin-sized pustules arranged in a peculiar form on the face, which disappeared spontaneously within one week precisely; bacteriological, mycological and virological tests were negative.

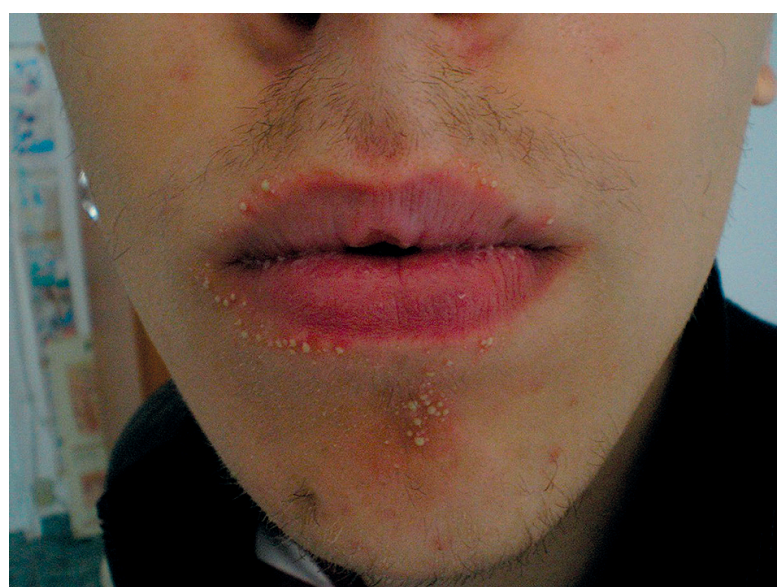

Figure 2. Patient 2. Small pustules (pin-sized), non-follicular, around the mouth 
These cases require the differentiation of:

1. Gram-negative folliculitis (GNF). The GNF is a hair follicle infection by Gram-negative organisms that can occur as a complication in patients receiving prolonged treatment with antibiotics for the therapy of acne vulgaris and rosacea. The pustules are follicular $[1,2]$.

2. Malassezia folliculitis (MF). Favorable external conditions, which are largely due to high temperature and humidity, and endogenous factors, such as immunosuppression, cause lipophilic yeast multiplication in the hair follicles [3]. The MF is a polymorphic dermatomycosis characterized by the development of small papules and pustules. In severe cases, molluscoid comedonal papules are typical.

3. Pustular psoriasis-like tinea incognito (TI). The TI is a dermatophytic infection in which topical or systemic steroids have modified the clinical appearance of the mycosis, mimicking other skin diseases [4].
In our patients simple hygiene methods were recommended and the lesions disappeared without any traces within 7 days. Recurrences have not been observed so far.

\section{References}

1. Sierra-Téllez D., Ponce-Olivera R.M., Tirado-Sánchez A., Hernández M.A., Bonifaz A.: Gram-negative folliculitis. A rare problem or is it underdiagnosed? Case report and literature review. N Dermatol Online 2011, 2, 135-138.

2. Gupta A.K., Batra R., Bluhm R., Boekhout T., Dawson T.L.: Skin diseases associated with Malassezia species. J Am Acad Dermatol 2004, 51, 785-798.

3. Brzeziński P., Kaczmarek D.: Malassezia folliculitis on the neck. N Dermatol Online 2010, 1, 22-25.

4. Kim W.J., Kim T.W., Mun J.H., Song M., Kim H.S., Ko H.C., et al.: Tinea incognito in Korea and its risk factors: nine-year multicenter survey. J Korean Med Sci 2013, 28, 145-151.

Anca Chiriac ${ }^{1}$, Piotr Brzeziński ${ }^{2}$ ${ }^{1}$ Department of Dermato-Physiology, Apollonia University Iasi, Iasi, Romania ${ }^{2}$ Dermatological Clinic, $6^{\text {th }}$ Military Support Unit, Ustka, Poland

ADDRESS FOR CORRESPONDENCE: Piotr Brzeziński MD, PhD

Dermatological Clinic $6^{\text {th }}$ Military Support Unit os. Lędowo $1 \mathrm{~N}$ 76-270 Ustka, Poland Phone: +4869211516 E-mail: brzezoo@wp.pl 